\begin{tabular}{|c|c|}
\hline \multicolumn{2}{|c|}{ Access this article online } \\
\hline \\
\hline
\end{tabular}

\title{
Consensus Statement on the use of cyclosporin A in patients with severe keratitis in dry eye syndrome in ophthalmology, rheumatology and hematology*
}

The Consensus Statement has been developed by a group of clinical experts in the fields of ophthalmology, rheumatology and hematology including (names in alphabetical order):

\section{Leszek Borkowski ${ }^{1}$, Dariusz Dobrowolski ${ }^{2}$, Lidia Gil ${ }^{3}$, Piotr Głuszko ${ }^{4}$, Iwona Grabska-Liberek ${ }^{5}$, Marta Misiuk-Hojło ${ }^{6}$, Ewa Mrukwa-Kominek ${ }^{7}$, Agnieszka Piekarska ${ }^{8}$, Joanna Przeździecka-Dołyk ${ }^{6,9}$, Bożena Romanowska-Dixon ${ }^{10}$, Jacek P. Szaflik" ${ }^{11}$, Bożena Targońska-Stępniak ${ }^{12}$, Monika Udziela ${ }^{11}$, Piotr Wiland ${ }^{13}$}

'Director's plenipontiary for Drug Analysis and Management in Wolski Hospital, President of the foundation "Razem w Chorobie" Board ${ }^{2}$ Department of Ophthalmology with Pediatric Unit, St Barbara $5^{\text {th }}$ Regional Hospital in Sosnowiec, Poland ${ }^{3}$ Department of Haematology and Bone Marrow Transplantation, Poznan University of Medical Sciences, Poznan, Poland ${ }^{4}$ Department of Rheumatology, National Institute of Geriatrics, Rheumatology and Rehabilitation, Warsaw, Poland ${ }^{5}$ Department of Ophthalmology, Medical Centre for Postgraduate Education, Warsaw, Poland ${ }^{6}$ Department of Ophthalmology, Wroclaw Medical University, Wroclaw, Poland ${ }^{7}$ Department of Ophthalmology, School of Medicine in Katowice, Medical University of Silesia, Katowice, Poland ${ }^{8}$ Department of Hematology and Transplantology, University Clinical Center, Medical University of Gdańsk, Gdańsk, Poland ${ }^{9}$ Department of Optics and Photonics, Faculty of Fundamental Problems of Technology, Wroclaw University of Science and Technology, Wroclaw, Poland

${ }^{10}$ Department of Ophthalmology and Ocular Oncology, University Hospital in Krakow, Poland

"Department of Ophthalmology, Medical University of Warsaw, Public Ophthalmic Teaching Hospital, Warsaw, Poland

${ }^{12}$ Department of Rheumatology and Connective Tissue Diseases, Medical University of Lublin, Lublin, Poland

${ }^{13}$ Department of Rheumatology and Internal Medicine, Wroclaw Medical University, Wroclaw, Poland

KEY WORDS: keratitis, dry eye syndrome, cyclosporin A.

\section{CONTENT OF THE CONSENSUS STATEMENT}

In December 2019, clinical experts in the fields of ophthalmology, rheumatology and hematology held a meeting in order to develop guidelines for the prevention and treatment of severe forms of dry eye syndrome (DES). During the meeting, an extremely important therapeutic problem was highlighted; namely the lack of patient access to reimbursed cyclosporin A. In light of above, a Consensus Statement was drawn up regarding the unavailability of sight-saving therapy in the form of reimbursed cyclosporin A to patients with severe DES.

The Consensus Statement was developed with references to the latest guidelines for the treatment of DES, including Polish Ophthalmological Society 2018 [1], TFOS DEWS II

\footnotetext{
${ }^{\star}$ Guidelines of scientific societies and associations (including the Polish Ophthalmological Society) do not constitute binding laws and do not determine the only correct procedures; they are only an opinion of a group of experts from a given field. The opinion reflects the current state of knowledge based on available scientific research results.

The guidelines do not exempt healthcare workers from personal liability with regard to making the correct decisions for individual patients.

Personal responsibility for the used therapeutic methods rests with all individuals who practise medicine. It should be based on thorough knowledge and practical skills, while observing necessary safety measures with regard to oneself and the patient.

Readers of this paper are obliged to make themselves familiar with current information on the presented treatments and pharmacotherapies with special attention paid to manufacturers' information on doses, time, and administration as well as side effects of the used drugs.

The publishers and editors of the paper shall not be responsible for any damages that could in any way be connected to the contents of this paper.
} 
[2-5], AAO 2018 [6], EULAR 2020 [7], EBMT 2019 [8], and the publication "Prevention and Treatment of Severe Keratitis in Dry Eye Syndrome" [9]. During the development of the experts' position, the opinion of dr. $n$. farm. Leszek Borkowski was taken into account in the section addressing the safety of medicinal products with cyclosporin [10].

Dry eye syndrome is a complex disorder characterized by inflammation, and instability and hyperosmolarity of the tear film. Severe forms of DES can lead to significant damage to the structures of the ocular surface (including severe keratitis), and neurosensory abnormalities.

Even though DES significantly reduces the quality of life of patients, it tends to be trivialized as a public health problem, despite the condition being one of the most common reasons to see an ophthalmologist. When providing their medical history, patients typically report a range of persistent symptoms including burning sensation, discomfort, itching, visual disturbances, irritation, photosensitivity, gritty feeling in the eyes, excessive tearing, and difficulty reading, driving, and working at the computer. The seriousness of the health problem is reflected in the fact that the lack of patient access to reimbursed treatment contributes to progression of the disease, ultimately resulting in ocular damage. The longer the period from the onset of DES symptoms to the diagnosis, the higher the severity of daily discomfort and pain, and the greater the adverse impact of the disease on the overall quality of life and professional activity of patients. The negative effect of moderate to severe DES on the quality of life of affected individuals is comparable to that of conditions requiring dialysis or severe angina.

Patients with DES feel distress related both to physical aspects caused by the specific nature of the disease (pain, disability) and mental health issues. Impaired mental well-being experienced by patients is caused, to a considerable extent, by the physical symptoms of the condition. At the same time, however, psychosomatic symptoms (depression, stress or anxiety), may significantly affect the subjective perception of ocular symptoms and pain, thus creating a vicious circle. Studies show that patients with DES are affected by higher levels of anxiety and depressive symptoms compared to individuals without the disease. Also, there is a confirmed relationship between the severity of DES and the degree of anxiety and depressive symptoms. Clinically, a greater severity of these conditions has been found in patients with severe forms of DES, e.g. due to Sjögren's syndrome (SS) [11].

It needs to be noted that DES is also associated with a significant economic burden both for patients and the society at large. The direct costs of the disease are related to appointments with medical specialists, diagnostic tests, and pharmacological and non-pharmacological therapies provided to patients. The indirect costs of DES are due to sickness absenteeism and ineffective work performance.

In light of the above, key importance should be attached to the correct diagnosis of the disease and the initiation of effective treatment - cyclosporin A in adult patients with severe keratitis accompanying DES who experience no im- provement despite using artificial tear products. A key focus must be placed on applying an interdisciplinary approach in the diagnostic and therapeutic process, as severe keratitis in DES is most typically encountered in patients with SS, and in patients with ocular graft-versus-host disease (GVHD).

In therapeutic practice, patients with severe keratitis in DES eye syndrome are most commonly treated with:

- corticosteroids,

- cyclosporin in the form of compounded eye drops or ointments,

- finished drug products containing cyclosporin A.

Long-term use of topical corticosteroids is associated with the risk of elevated intraocular pressure, cataract or opportunistic ocular infections. Consequently, drugs of this group must be used for a short time only, which fails to break the vicious cycle of inflammation in a proportion of patients. In contrast, topical treatment with cyclosporin A can be used on a long-term basis, and is capable of controlling inflammation, but the lack of reimbursed cyclosporin A significantly reduces patient access to effective therapy.

Currently, Ikervis is the only product in the form of eye drops containing cyclosporin A which is approved for marketing in the European Union. Prior to the approval of Ikervis, Restasis (a drug approved in the USA) was imported and reimbursed in the target import procedure. Ikervis is an emulsion containing $0.1 \%$ cyclosporin. Unlike Restasis, which is an anionic castor oil-in-water emulsion, Ikervis has the form of cationic oil-in-water emulsion. As a result, cyclosporin remains as tear film on the ocular surface for longer, which increases the bioavailability of the drug.

Unlike corticosteroids, cyclosporin A can be used on a long-term basis. According to the Summary of Product Characteristics, response to treatment should be assessed at least every 6 months.

It is important to note that Ikervis is currently listed in drug reimbursement schemes in most European countries (even in Croatia, the Czech Republic, Slovakia, Slovenia and Hungary), while in Poland it is excluded from public funding. The exclusion of Ikervis from the drug reimbursement scheme, combined with the concurrent loss of access to Restasis reimbursed under the target import procedure, has significantly worsened the situation of patients in Poland.

Cyclosporin A is a cyclic immunomodulatory polypeptide with immunosuppressive activity. It has antiinflammatory properties and inhibits cellular immune responses. In addition, cyclosporin blocks the synthesis and/or release of proinflammatory cytokines, including interleukin 2 (IL-2), also known as T-cell growth factor (TCGF). Another effect induced by cyclosporin A is an increased release of antiinflammatory cytokines. The antiinflammatory activity of cyclosporin justifies its use in patients with moderate to severe DES which is a condition with an underlying immune-mediated inflammatory mechanism. Cyclosporin in the form of eye drops reaches T lymphocytes in the cornea and conjunctiva, where it deactivates calcineurin phosphatase. The inactivation of calcineurin prevents the translocation of the 
NF-AT transcription factor into the cell nucleus. Through this mechanism, cyclosporin blocks the release of proinflammatory cytokines.

A review of adverse effects, special warnings and precautions as well as drug interactions points to the therapeutic importance of cyclosporin regardless of the type of clinical application. An absolute recommendation under the current pharmacological doctrine is that patients in such cases should be treated with drugs manufactured under GMP supervision, by recognized pharmaceutical manufacturers, and controlled by the marketing authorization holder and competent national and EU regulatory authorities.

Therefore, it should be emphasized that cyclosporin in the form of a compounded drug is not an alternative to the finished drug product because:

- eye drops prepared in pharmacies are known to be of inferior quality to those made industrially (pharmacies have no infrastructure to consistently ensure appropriate classes of air and environmental cleanliness; no drug formulation facilities with "dirty" and "clean" man locks; and often no separation of works performed in the preparation of standard and aseptic formulations),

- pharmacies prepare cyclosporin A eye drops using available pharmacopeia-approved vehicles (liquid carriers). However, their composition may deviate from the manufacturer's specifications, resulting in limited bioavailability, and hence problems with achieving therapeutic concentrations of ophthalmic cyclosporine, depending on the manufacturing site,

- compounded drugs prepared in pharmacies are not evaluated for proper dosage in clinical studies.

Consequently, the experts' conclusion is that the finished drug product shows superior quality, efficacy, stability and safety to pharmacy-made drugs, which is why substitution is not recommended. Cyclosporin as an API is not suitable for such substitution because of its narrow therapeutic index, nonlinear pharmacokinetics, and the risk of deviations in bioequivalence indicated above. A similar position was expressed in the recommendation of September 2019 issued at the request of the Minister of Health by the Dutch National Health Care Institute (Zorginstituut Nederland) [12].

Finished drug products have an advantage over compounded drugs in the following aspects:

- no designated MAH,

- no dedicated procedure for reporting adverse effects of compounded drugs,

- no pharmacovigilance system for the safety of use of compounded medicinal products,
- no system for managing risks associated with using compounded medicinal products,

- no intra-pharmacy control of the risk of falsification of the API or pharmacopeial raw material,

- no release of compounded drug batch by a qualified person, as there are no batches, and each drug may be different,

- no patient information leaflet and summary of product characteristics approved by a competent authority.

Moreover, since more and more pharmacies restrict the compounding of ophthalmic drugs, access to pharmacies where a compounded drug with cyclosporin can be ordered is becoming increasingly difficult. Since a finished drug product is commercially available, measures should be taken to include the product in the drug reimbursement scheme.

In cases involving adverse effects of a compounded drug, the option to perform a follow-up qualitative study under the pharmacovigilance procedure is limited due to reasons related to the availability of the compounded drug after adverse effects are reported, and the possibility of qualitative and quantitative control of the drug.

Based on the above considerations, it should be concluded that the only recommended type of cyclosporin A are eye drops in the form of a finished drug product authorized for marketing in the EU.

In Poland, there are interdisciplinary guidelines for the treatment of severe forms of DES developed in cooperation with ophthalmologists, rheumatologists and hematologists ("Prevention and Treatment of Severe Keratitis in Dry Eye Syndrome") which clearly recommend the use of cyclosporin $A$ in the form of a finished drug product as an effective and safe therapeutic option in the analyzed patient population. However, the guidelines cannot be implemented in clinical practice because of reimbursement restrictions (Ikervis is excluded from the reimbursement scheme). As a result, a growing group of patients is deprived of access to treatment, even though the product is reimbursed from public funds in the majority of EU member states.

Taking into consideration all the arguments presented in this document, experts in the fields of ophthalmology, rheumatology and hematology agree that there is a strong unmet need to integrate cyclosporin A into clinical practice in Poland. Consequently, it is recommended that measures are taken to ensure that medically necessary treatment is available to patients. To achieve this, a finished drug product containing cyclosporin A should be added to the drug reimbursement scheme.

\section{DISCLOSURE}

The authors declare no conflict of interest. 


\section{References}

1. Wytyczne Polskiego Towarzystwa Okulistycznego dotyczące diagnostyki i leczenia Zespołu Suchego Oka, PTO_Wytyczne_Zespol_ Suchego_0ka_2018.PDF

2. Craig JP, Nichols KK, Akpek EK, et al. TFOS DEWS II definition and classification report. Ocul Surf 2017; 15: 276-283.

3. Stapleton F, Alves M, Bunya VY, et al. TFOS DEWS II epidemiology report. Ocul Surf 2017; 15: 334-365.

4. Wolffsohn JS, Arita R, Chalmers R, et al. TFOS DEWS II diagnostic methodology report. Ocul Surf 2017; 15: 539-574.

5. Jones L, Downie LE, Korb D, et al. TFOS DEWS II management and therapy report. Ocul Surf 2017; 15: 575-628.

6. American Academy of Ophthalmology, Dry Eye Syndrome Preferred Practice Pattern, 2018.

7. Ramos-Casals M, Brito-Zerón P, Bombardieri S, et al. EULAR recommendations for the management of Sjögren's syndrome with topical and systemic therapies. Ann Rheum Dis 2020; 79: 3-18.

8. Inamoto $Y$, Valdés-Sanz N, Ogawa Y, et al. Ocular graft-versus-host disease after hematopoietic cell transplantation: Expert review from the Late Effects and Quality of Life Working Committee of the CIBMTR and Transplant Complications Working Party of the EBMT. Bone Marrow Transplant 2019; 54: 662-673.

9. Wytyczne PTO, PTR, PTHiT dot. zapobiegania i leczenia ciężkiego zapalenia rogówki w przebiegu ZSO. Retrieved from https://www. pto.com.pl/aktualnosci/wytyczne-pto-ptr-pthit-dot-zapobiegania-i-leczenia-ciezkiego-zapalenia-rogowki-w-przebiegu-zso.

10. Borkowski L. Opinia w sprawie możliwości zastąpienia produktu leczniczy lkervis /jedyne krople do oczu z cyklosporyną A dopuszczone do obrotu na terenie Unii Europejskiej/ preparatami recepturowymi zawierającymi cyklosporynę A. Retrieved from http://www.fundacjarazemwchorobie.pl/uploads/0pinia\%20leki\%20oczne\%20z\%20cyklosporyn\%C4\%85.pdf

11. Le $Q$, Zhou $X, G e L$, et al. Impact of dry eye syndrome on vision-related quality of life in a non-clinic-based general population. BMC Ophthalmol 2012; 12: 22.

12. Zorginstituut Nederland, Ciclosporin (Ikervis ${ }^{\circ}$ ) for the treatment of severe keratitis in adult patients with the dry eye syndrome. Retrieved from https://english.zorginstituutnederland.nl/binaries/zinleng/documents/reports/2019/09/18/ciclosporin-ikervis-for-the-treatment-of-severe-keratitis-in-adult-patients-with-the-dry-eye-syndrome 